\title{
Wie kommt ein Kind zu einem Schatz an Worten?
}

\section{Patienteninformation}

Erinnern Sie sich, als Sie jemanden in einer fremden Sprache reden hörten und nichts verstehen konnten? Genauso ergeht es kleinen Kindern, die ganz am Anfang des Spracherwerbs stehen. Eigentlich haben sie es noch schwerer, da sie zunächst noch nicht einmal wissen, dass überhaupt gesprochen wird oder was „Sprechen“ bedeutet. Es existieren unterschiedliche Theorien darüber, wie Kinder innerhalb von nur wenigen Jahren vom Nichtverstehen zu einem Schatz an Worten kommen. Im Folgenden wird die Annahme vertreten, dass Kinder Wörter in sozialen Kontexten lernen, indem sie die kommunikativen Absichten des Gegenübers verstehen. Aber was bedeutet das?

\section{Das erste Wort \\ $\nabla$}

Im Laufe des ersten Lebensjahres fängt das Kind an, sich für seine Umwelt zu interessieren. Es betrachtet zum Beispiel sehr aufmerksam die Rassel, die es in der Hand hält, oder das freundliche Gesicht, das sich über es beugt. Zu Beginn widmet es seine Aufmerksamkeit noch entweder der Rassel oder dem Gesicht, nicht aber beidem. Erst im Alter von 9-12 Monaten gelingt es dem Kind, 2 Dinge in den Fokus seiner Aufmerksamkeit zu nehmen. Dieser Entwicklungsschritt wird auch als 9-Monats-Revolution bezeichnet. Es folgt vielleicht dem Blick des Erwachsenen und entdeckt die Rassel. Es schüttelt sie und blickt zurück zum Erwachsenen, um zu sehen, ob er auch lacht. Jetzt verfolgen Kind und Erwachsener dieselbe Absicht: ein Spiel mit der Rassel. Typischerweise begleiten wir solche Situationen mit Worten oder Sätzen (z.B. „Da ist die Rassel.“ „Eine rote Rassel!“ „Ist die schön, die Rassel?“). Und typischerweise wiederholen sich solche Situationen - vielleicht gleich am nächsten Tag. Solche wiederkehrenden Situationen, in denen das Kind die Absicht des Erwachsenen versteht und auch teilt und in denen bestimmte Wörter immer wieder auftauchen (z.B. „Rassel“), bil- den die Basis dafür, dass ein Kind zu einem ersten Verständnis von Sprache kommt und auch Sprache gebraucht. So ist es nicht verwunderlich, wenn die meisten Kinder in der Zeit des ersten Geburtstages ihr erstes Wort sprechen. Das erste Wort bezeichnet oft eine Person in der direkten Umgebung („Mama“), einen Gegenstand, den das Kind liebt („Ball“), oder eine Tätigkeit, die es immer wieder ausführt („weg“ für verstecken). Kinder verwenden also zunächst Wörter, die eine Person oder etwas bezeichnen, mit der oder dem sie viel Erfahrung gemacht haben.

\section{Schatz an Worten}

In der Folgezeit erweitert sich der kindliche Wortschatz mitunter erheblich, und zwar um viele neue Wörter und Wortarten. Es kommen Tätigkeitswörter wie „essen“ oder „malen“ hinzu. Das Kind lernt Eigenschaftswörter wie „heiß“ oder „schmutzig“ und Funktionswörter wie „da“ oder „die“. Es geht weiter darum, dass das Kind wiederkehrende Situationen erlebt, in denen es die Absicht des Gegenübers versteht und so den Schatz an Worten entdeckt, den solche Situationen hervorbringen.

In einer ersten Normierungsstudie an der Universität Oldenburg haben gut 1200 Eltern mit dem Elternfragebogen FRAKIS den Wortschatz ihrer anderthalb- bis zweieinhalbjährigen Kinder eingeschätzt. Die Ergebnisse bestätigen, was wir aus anderen Sprachen schon wissen. Der Wortschatz von individuellen Kindern, die gleich alt sind, ist sehr unterschiedlich groß. Es scheint normal, wenn Eltern für ihr zweijähriges Kind einen Schatz an Worten zwischen 49 und 456 Wörtern angeben. Die Eltern haben dabei aus einer Liste von 600 Wörtern gewählt. Das ist eine enorme Spanne und bedeutet, dass nicht ein bestimmter Wortschatzwert richtig ist, sondern die Unterschiedlichkeit normal ist.

\section{Vokabelspurt \\ $\nabla$}

Viele Eltern beschreiben für ihr Kind, dass sich ab einem bestimmten Zeitpunkt der Wortschatz sprunghaft erweitert. Dies nennt man den Vokabelspurt. Meist ist das Kind zwischen 17 und 24 Monate alt und der Spurt setzt ein, wenn der Wortschatz zwischen 50 und 100 Wörter umfasst. Entgegen der weitläufigen Meinung tritt der Vokabelspurt aber nicht bei allen Kindern ein. Dies bedeutet auch keinen Nachteil und steht auch nicht automatisch für ein Risiko für eine mögliche Sprachstörung. Manche Kinder erweitern ihren Schatz an Worten einfach nach und nach oder in kleinen Sprüngen.

\section{Fazit}

Kinder brauchen viele gemeinsame, wiederkehrende Erlebnisse, in denen gesprochen wird. Es ist ganz normal, dass sie ihren Schatz an Worten in unterschiedlichem Tempo erweitern. Ob das Tempo zu langsam und der Wortschatz eines Kindes zu klein ist, darüber kann nicht an einem einzigen Tag oder mit einem einzigen Fragebogen entschieden werden.

\section{Dr. rer. nat. Barbara Stumper, Oldenburg}

Literatur

1 Szagun G, Stumper B, Schramm SA. FRAKIS Fragebogen zur frühkindlichen Sprachentwicklung. Frankfurt: Pearson; 2009

2 Tomasello M. Constructing a Language: A Usage-Based Theory of Language Acquisition. Harvard: University Press; 2005

Weitere Literatur bei der Verfasserin. barbara@stumper.de

überreicht durch Thieme und 